\title{
LA FUNCIÓN DE LOS CONSTRUCTORES DE ESPACIO MENTAL EN EL DISCURSO PUBLICITARIO: EL USO DE LA SEGUNDA PERSONA OBJETIVADORA TUU
}

\author{
María José SERRANO \\ Universidad de La Laguna (España)
}

\section{RESUMEN}

El discurso publicitario tiene como función primordial la persuasión para promover el consumo. Son muchas las formas lingüísticas que conducen a cumplir tal objetivo, entre las cuales destaca el uso de la segunda persona del singular $(t u ́)$, puesto que es la persona que más claramente indexa al interlocutor o audiencia. En este trabajo se analizará el uso de la segunda persona del singular tú con función objetivadora en el discurso publicitario, esto es, cuando la referencia no solo incluye al interlocutor o audiencia, sino, también, a la primera persona, que en el caso del discurso publicitario se entiende como participante primario o secundario. El significado objetivador de la segunda persona es gradual y en esa distinción los constructores de espacios mentales, tales como cuando, si y otros elementos temporales, resultan determinantes. La ausencia de constructores promueve el significado objetivador, mientras que su presencia contribuye a la creación del valor que denominamos objetivador +. Estos significados se analizarán en diversos tipos de anuncios y ámbitos comunicativos. Los resultados indican que el significado objetivador de la segunda persona del singular $(t u ́)$ se construye con mayor frecuencia mediante la ausencia de constructores de espacios mentales en todos los tipos de texto y ámbito. En cambio, la presencia de constructores de espacios mentales es mayor en ciertos textos y ámbitos, con lo cual el contenido se presenta de forma general o universal.

PALABRAS CLAVE: constructores de espacio mental; objetivación; publicidad; segunda persona del singular tú; variación morfosintáctica.

* Este trabajo forma parte del proyecto de investigación «Formalización e integración de dimensiones estilísticas en la morfosintaxis funcional y cognitiva del español: la desubjetivación gramatical y el punto de vista del discurso». Se agradece a la Universidad de La Laguna (ULL) y al Ministerio de Ciencia, Innovación y Universidades la ayuda concedida. Asimismo, esta publicación es parte del proyecto de I+D+i/ con referencia PID2020-113474GBI00, financiado por el Ministerio de Ciencia e Innovación/Agencia Estatal de Investigación/10.13039/ 501100011033 . 


\section{AbSTRACT}

As it is known, the main goal of advertising discourse is to promote the acquisition of products. To accomplish such a goal, the use of linguistic forms is determinant. One of these forms is the second-person singular $t u$, as it is the form that most clearly indexes the interlocutor or audience. This paper will analyze the second-person singular tú which does not only include the interlocutor or audience, but also the participant speaking or writing. In mass media communication, such a participant may be primary or secondary. This use has been labelled as objectivizing, since it implies a displacement from the first person to the second one. By means of the presence or absence of spacebuilders such as cuando, si or others, this meaning is gradual; it may be more personal or closer to the speaker (objectivizing) or it may be presented as a fact universally shared (objectivizing + ). The absence of any spacebuilder gives rise to the plain objectivizing meaning, while their presence will imply a step forward and mark the reference as objectivizing + . These variants will be studied in different types of advertisements and communicative areas. The results indicate that the objectivizing variant is the most frequent one in advertising discourse across all the types of texts and communicative areas analyzed. However, the use of spacebuilders is common in certain texts and areas, such as advertising spots and social media posts, among others, thus presenting the content as ultimately more objective.

KEYWORDS: spacebuilders; objectivity; advertising; second-person singular tú; morphosyntactic variation.

Fecha de recepción: 19/03/2021

Fecha de aceptación: 06/05/2021

Fecha de la versión definitiva: 18/05/2021

\section{El Discurso PUblicitario}

Suele considerarse que el discurso publicitario forma parte de lo que se denomina «discurso de los medios de comunicación» o, más concretamente cuando se refiere a la lengua española, «español en los medios de comunicación». Esta denominación hace referencia a una técnica histórica del lenguaje; a una lengua en concreto, el español, tal y como se produce en los medios de comunicación colectivos o sociales (Romero Gualda 1996: 9). Además, alude a un uso especial del código lingüístico en esta situación comunicativa, que normalmente lleva aparejada una connotación negativa mediante su errónea consideración como «lenguaje sectorial», «jerga»o «variedad marginal del español» (Romero Gualda 1996: 9-10).

A pesar de que hay determinadas formas lingüísticas que ya se consideran prototípicas de los medios de comunicación por su alta frecuencia en determinados ámbitos, en general, los usos y variantes presentes en el discurso de los medios de comunicación no se diferencian de los que apare- 
cen en otros discursos tanto orales como escritos. Se tiende a englobar, además, todos los tipos de textos y géneros discursivos bajo la denominación de «medios de comunicación», sin que se haya prestado una especial atención a cada uno de estos tipos de forma independiente.

Con todo, por lo que respecta a la publicidad, se trata de una práctica social completamente asentada en casi todas las sociedades y puede adoptar un rol modelizador. Puede ser un estímulo, un signo plural o un texto que introduce, en cada caso, focos de contemplación no totalmente coincidentes (Vázquez Medel 1993: 50). Al tratarse de una comunicación específica (Dyer 1982), resulta necesario considerarla como un tipo especial de discurso, dotado de unas características comunes (Coleman 1990; Cook 2001: 3-4), el cual está indudablemente basado en el desarrollo de la persuasión (Hermeren 1999; Vestergaard y Schroder 1985). En el discurso publicitario confluyen, como es sabido, el sistema lingüístico y otros elementos paralingüísticos e icónicos, que indudablemente terminan por conformar el significado del mensaje. Participa, por lo tanto, de una heterogeneidad de códigos (Romero Gualda 1996: 16) que condiciona la interpretación y el análisis del propiamente verbal.

Todos los elementos lingüísticos textuales y paratextuales de la publicidad están impregnados de la función persuasiva, la cual está orientada al consumo de un producto o a la adhesión a una idea. La función de la persuasión pretende convencer al receptor sobre un tema o idea específico utilizando la argumentación como soporte principal y fundamental (Fuentes Rodríguez y Alcaide Lara 2002: 16). Por lo tanto, «al configurar su mensaje, el publicista, y la empresa o institución para la que diseña el anuncio, ejercen un estricto control sobre todos sus aspectos lingüísticos y no lingüísticos con vistas al logro de tales objetivos» (Aijón Oliva 2009: 2).

Según Ferraz Martínez (1993: 28), la persuasión se produce mediante dos vías paralelas: la implicación de los receptores y la exaltación y ponderación de los productos. La implicación del destinatario como posible consumidor se consigue mediante diversos recursos emocionales; no en vano la persuasión en la publicidad está inevitablemente basada en el fomento de la emoción y, con ello, en el objetivo de asegurar una respuesta positiva del consumidor (Hernández Toribio 2006: 89-94). Dicho de otro modo, la persuasión emocional pretende llegar al corazón de dicho consumidor -concebido como audiencia, pero generalmente singular- y crea en él la ilusión de sentirse único. En definitiva, la persuasión busca sensibilizar a los consumidores mediante estrategias que apelan a las emociones (Hernández Toribio 2006: 227).

La publicidad presupone la existencia de un consumidor (oyente o lector) al que se le considera como referente (Goddard 1998: 32; Zacher 1967), de tal forma que el uso de los pronombres personales se erige como 
una categoría destacada, incluso recomendable, en este tipo de discurso (Armstrong 2010: 193). Así pues, es fácil predecir que en el discurso publicitario se produzcan diversas formas de aludir a la figura del destinatario. La más recurrente y quizás más esperable de ellas es el uso de la segunda persona del singular ( $t u$ ), la cual ha sido ya objeto de estudio en este tipo de discurso (Hernández Toribio 2006; Serrano y Hernández Toribio 2015).

En esta investigación profundizaremos en el uso de los referentes personales de las formas pronominales como recursos de persuasión en el discurso publicitario. En concreto, se abordará una de las formas menos estudiadas del paradigma pronominal en este tipo de texto: el uso de la segunda persona del singular $(t u ́)$ cuando no indexa realmente a un referente de segunda persona, sino a la esfera referencial del propio hablante, que, en el caso del discurso publicitario, constituye en última instancia la voz de la empresa anunciadora. El significado de esta persona gramatical utiliza la forma de la segunda persona del singular $(t u ́)$ para incluir en la referencia a la persona que habla o escribe, por lo tanto, como se verá, el significado deja de ser deíctico y se transforma en objetivador (Aijón Oliva y Serrano 2014; Serrano 2013; Serrano y Aijón Oliva 2012), exhibiendo una amalgama referencial de la primera y segunda persona. Partimos de la idea de que las personas gramaticales son construcciones cognitivas y pragmáticas que, en principio, pueden aludir a casi cualquier referencia extradiscursiva (Aijón Oliva 2019: 84; 2020: 5). A partir de su significado inherente, cada persona construye discursivamente la referencia contextual de una determinada manera.

La persona gramatical objeto de estudio se analizará desde una perspectiva discursivo-cognitiva; por una parte, se considerará esta forma como una elección de significado específico, no asimilable ni alternante a otras opciones (Serrano 2016) y, por otro, se analizarán los elementos que contribuyen a la creación de dicho significado en el discurso publicitario en el marco de la teoría de los espacios mentales.

\section{LAS PERSONAS EN LA PUBLICIDAD}

El discurso publicitario presenta de forma casi categórica una interacción entre personas, la cual, en realidad, pretende recrear una necesaria interacción entre la empresa publicitante y el consumidor potencial. Como indica Leech (1966: 34), existen dos tipos de escenarios en la publicidad. El primero contiene participantes primarios o interlocutores directos, en el cual el publicitante indexa al interlocutor o audiencia de forma directa. Se trata de aquellos anuncios (orales o escritos) en donde el participante primario es la empresa publicitante, que se dirige directamente a 
la audiencia, a la que indexa como segunda persona. Los siguientes mensajes publicitarios (1) y (2), son ejemplo de ello.

(1) Silla salvaescaleras Stannah. Tu libertad de movimiento sin barreras. Al instalar una silla salvaescaleras Stannah podrá hacer su escalera segura otra vez, disfrutar de toda su casa, sin dolores, sin inseguridad, y todo esto sin tener que cambiar su rutina. ( $\mathrm{CIE}<\mathrm{Adv} 2021-1>$ )

(2) ¿Ya has pensado qué le regalarás a papá el Día del Padre? Tenemos el mejor regalo para él. Entérate de cómo conseguirlo la próxima semana en Pronto. (CIE<Adv2021-2>)

El segundo escenario es aquel que introduce participantes secundarios, que actúan como consumidores para publicitar el producto o la idea de forma directa o indirecta. El primer caso se observa en el siguiente ejemplo (3), en el que una persona conocida promociona el producto cediendo su voz y su imagen a la marca que se publicita.

(3) Mi pelo es una de mis señas de identidad, por ello lo que persigo es mantenerlo sano y con un aspecto suelto y con movimiento. Mis infalibles son el acondicionador AquaLight de Pantene Pro-V -que uso siempre que me lavo el pelo para mantenerlo hidratado sin sacrificar la sensación de ligereza- y la mascarilla nutritiva de la misma colección que aplico una vez por semana. (CIE<Adv2021-3>)

Tras el siguiente diálogo de dos personas anónimas, una voz en off introduce el producto publicitado. Los participantes secundarios actúan de forma indirecta en la promoción del producto, como se observa en (4).

(4) A: Tengo que decirte una cosa

B: ¿Qué pasa?

A: Estoy saliendo con Marina

B: ¿Marina?

A: Tu exnovia

Voz en off: La amistad se basa en la comunicación, nuevo Seat Ibiza con tecnología full ink. (CIE<Adv2017-371)

Si bien los participantes secundarios suelen ser personajes con una cierta relevancia social que actúan como autoridades, y que, mediante sus intervenciones, abundan en las bondades del producto o idea publicitada (como en el ejemplo (3)), estos también pueden actuar como terceras personas a las que se alude, porque supuestamente han validado el producto o idea mediante su experiencia (naturalmente positiva), sobre dicho producto o idea. Este último modo de publicidad se ha denominado publicidad de indexación indirecta (Barron 2012: 174-175; Leech 1966: 34-35). Véase el ejemplo (5). 
(5) Massumeh. La marca de las celebrities ha velado por la belleza de Penélope Cruz, Isabel Preysler, Noor de Jordania o Farah Diba, entre otras. ¿Por qué no dejas que lo haga por la tuya? Tú también te lo mereces. (CIE<Adv2021-4>)

La función persuasiva de los mensajes publicitarios puede manifestarse también mediante la presentación de personajes anónimos en ellos, con el objeto de presentar el producto o idea como algo accesible y necesario para el conjunto de consumidores. El ejemplo (6), formulado en tercera persona, es una muestra de cómo el anunciante valida la bondad del producto mediante la utilización de un consumidor anónimo, con el cual se puede identificar cualquier individuo.

(6) Marina, que no es partidaria de las medicinas, confía en Vitaldona porque sabe que «es un producto totalmente natural», que puede encontrar en cualquier farmacia. ¿Tú también quieres sentirte bien?

$(\mathrm{CIE}<\mathrm{Adv} 2021-4>)$

Como se observa claramente, en todos los mensajes publicitarios participan personas que constituyen las destinatarias del consumo. Sus referentes se indexan predominantemente en segunda persona, aunque ello no impide que en los anuncios aparezcan también, aunque, en menor medida, referentes de primera y tercera persona. Por lo tanto, el uso del paradigma de las formas pronominales es recurrente y su análisis se hace más que necesario para la correcta interpretación del mensaje publicitario, así como para desentrañar el funcionamiento de la persuasión que define a este tipo de texto.

\subsection{La seguna persona del singular (tú) en el discurso publicitario}

Como cabía esperar, la segunda persona del singular tú es una de las formas más frecuentes en el discurso publicitario; si el objetivo del discurso publicitario es el de persuadir para la compra de un producto o adhesión a una idea, el referente de segunda persona singular resulta idóneo para construir el mensaje publicitario. Y ello pese a que el referente de esta persona singular es un conjunto plural de ellos o audiencia, generalmente inespecífica y desconocida. Sin embargo, el potencial referencial que posee la segunda persona del singular como estrategia de indexación no lo posee el plural, normalmente de naturaleza referencial más difusa en todas sus manifestaciones, tanto de primera como de segunda persona (Aijón Oliva 2019: 151-159, 190; Serrano 2020; Serrano y Aijón Oliva 2014b). El uso de esta forma refleja una intención del publicitante de acercamiento 
al consumidor de la misma forma que lo haría cara a cara en la conversación cotidiana (Serrano y Hernández Toribio 2015: 110) ${ }^{1}$.

El uso de la segunda persona del singular tú en la publicidad, en su significado prototípico que indexa al interlocutor o audiencia como potencial consumidor, se ha estudiado como un caso de variación morfosintáctica. Siguiendo la tendencia habitual de la lengua española, los resultados indicaron que la forma omitida es la más frecuente (Serrano y Hernández Toribio 2015: 119). El siguiente ejemplo (7), muestra un mensaje publicitario donde el referente de segunda persona se muestra como singular y el pronombre se omite.

(7) Todo lo que $\varnothing$ necesitas cuando $\varnothing$ lo necesitas. Alimentación y bebidas. Perfumería y droguería. Ocio y regalos. Sólo Opencor te sienta así de bien. Porque sólo en OpenCor $\varnothing$ compras lo que te gusta, como a ti te gusta. Más comodidad. Porque $\varnothing$ compras cuando $(\varnothing)$ te viene bien $[\ldots]$. (Telva885/en13)

Por su parte, la expresión del pronombre implica que el referente emerge en la escena comunicativa, lo cual conlleva que este quede realzado pragmáticamente y constituya el centro del mensaje publicitario, gracias a la informatividad que conlleva el sujeto expreso, como se observa en (8) (Serrano y Hernández Toribio 2015: 118). De hecho, este enunciado se ha convertido en el eslogan de esta marca de cosmética.

(8) Combate los signos de la edad en un gesto. El primer anti-edad de L'Oréal Paris. Porque tú lo vales. L'Oréal. (Telva874/feb12)

Sin embargo, esta forma no siempre adquiere un significado que indexa a la segunda persona del singular prototípica. En el ámbito publicitario, puede tener una referencia dual: puede aludir a alguien presente en el anuncio, o al propio consumidor (Cook 2001: 158-159). Como se dirige a una audiencia desconocida y ficticia, se considera también como segunda persona del singular tú inespecífica (Aijón Oliva 2019: 189), toda vez que la indexación no recae sobre un referente concreto y específico, como sí ocurre con la variante deíctica o específica (Aijón Oliva 2019: 189). Asimismo, puede incluir al participante que habla o escribe, a través de su propia voz, o bien a través de un consumidor ficticio presente en el anuncio. Esta referencia que incluye a la primera y a la segunda persona será la que se analizará en este trabajo.

\footnotetext{
${ }^{1}$ De hecho, este acercamiento se logra también mediante el uso de otros recursos de apelación directa, tales como enunciados interrogativos, enunciados exhortativos, vocativos, expresiones de referencia personal e interjecciones, entre otras (Hernández Toribio 2006: 69-118).
} 
2.2 La segunda persona del singular tú objetivadora. Los constructores de espacio mental

De modo muy frecuente y en cualquier situación comunicativa, el referente de esta persona no es una segunda persona, sino una representación de la primera, la cual, al mismo tiempo, puede incluir al interlocutor o al conjunto de referentes indeterminado. Nótense las diferencias entre los siguientes anuncios publicitarios construidos con la segunda persona del singular tú. En (9), el anuncio está referencialmente dirigido al consumidor, en este caso, el progenitor que dará la papilla a su hijo constituye el referente de la segunda persona.

(9) Con papillas Nestlé, SABES que le estás dando lo que necesita. SABES que los cereales son la base de una dieta equilibrada. SABES que no hay ningún otro alimento que proporcione la misma cantidad de nutrientes por toma. SABES que se digieren fácilmente. (CIE<Adv2021-5>)

En el ejemplo (10), el uso de la segunda persona, tanto en su forma de sujeto como de objeto (a excepción de la forma imperativa final, que induce a la interpretación deíctica del morfema te en la frase «te espera»), tiene como referente al publicitante, que utiliza la segunda para presentar el contenido como experiencia propia, y esta se transforma en consejo o instrucción consabida que se quiere compartir con la audiencia. Esta audiencia, por tanto, forma parte también de ese referente.

(10) ¿Sabes cuándo te das cuenta de que lo tuyo con Irene va en serio? no es cuando le dices «te quiero» ni al dejar tu cepillo en su casa, sabes que va en serio cuando te expulsan de «solterazos» y te agregan a parejitas, sí, sí, prepárate para todo lo que te espera. (CIE $<$ Adv2021-120>)

Debido a esa naturaleza referencialmente difusa o no deíctica del interlocutor, este uso de la segunda persona ha sido clasificado como tú impersonal, inespecífico o generalizador (Beinhauer 1968: 113; Cameron 1993; Enríquez 1984; Hidalgo Navarro 1996-1997; Kany 1969: 29; Kitagawa y Lehrer 1990; NGLE 2010: $\$ 16.2 u$; Seco 1989: 374, entre otros), y también como de discordancia deliberada (Gili Gaya 1976: 33), entre otros términos.

Los valores de inespecificidad y de generalización se adaptan de alguna forma al significado de este uso; sin embargo, está lejos de constituir una estrategia de impersonalización. Cualquier uso de la persona gramatical implica necesariamente una referencia y, de hecho, la segunda persona del singular $(t u ́)$ constituye una forma de representación difuminada de la primera persona; en otras palabras, podríamos parafrasear su significado como «cuando tú eres yo» (Serrano y Aijón Oliva 2012); es decir, se trata de un uso que incluye siempre al hablante (Aijón Oliva 2019: 192), el cual emplea 
la segunda persona para alejar el contenido expresado de su esfera personal y desplazar del punto de vista discursivo hacia la audiencia (Aijón Oliva 2020: 11). Con este cambio, el contenido se torna más objetivo que si se hubiese utilizado la primera persona del singular yo, por lo tanto, constituye un recurso de desubjetivación (Aijón Oliva 2020: 9). Ello es debido a que dicho cambio implica un descenso en la prominencia cognitiva del referente; el de la primera persona es más prominente o más referencialmente accesible que el de la segunda ${ }^{2}$. Por ello, y por el consecuente tránsito que implica de la primera persona hacia la segunda, este uso ha recibido la denominación de segunda persona objetivadora (Aijón Oliva y Serrano 2014; Serrano 2013, 2021a; Serrano y Aijón Oliva 2012) o inclusiva del hablante (Aijón Oliva 2019: 192). Mediante este uso, al interlocutor o audiencia se les invita directamente a imaginarse o a incluirse en la situación o evento descrito (Siewierska 2004: 212), y ello resulta muy útil para el desarrollo de la función persuasiva en la publicidad.

Con el objeto de establecer los elementos contextuales que apoyan la intepretación no deíctica de esta forma, los llamados inductores o activadores de genericidad han adquirido una relevancia especial en su análisis (Guirado 2011: 29; Hernanz Carbó 1990: 166; Hugo Rojas 2011: 167; Laberge y Sankoff 1979). Sin embargo, una investigación reciente demuestra que los elementos lingüísticos que coaparecen con la segunda persona objetivadora $(t u ́)$ son, en realidad, marcadores temporales de variada naturaleza (entre ellos cuando, si y otros), los cuales construyen un punto de vista temporalmente difuso y continuo que favorece la interpretación objetivadora (Serrano 2021b). Esto puede constituir un avance con respecto a apreciaciones como la de Fernández Soriano y Táboas Baylín (1999: 1734-1735), quienes consideran que un factor decisivo para el establecimiento del valor genérico es la ausencia de un punto temporal referencialmente concreto en la cláusula o en el contexto inmediato.

Los marcadores temporales que promueven esta interpretación de la segunda persona del singular tú se pueden analizar de una forma más precisa y efectiva en el marco de la teoría cognitiva de los espacios mentales (Fauconnier 1985 [1994]; Fauconnier 1997). El enfoque cognitivo tiene entre sus fundamentos el de demostrar que cualquier elección gramatical conlleva repercusiones en el significado (Evans y Green 2006: 744), por ello, la adopción de este enfoque ha beneficiado al estudio de la variación morfosintáctica en español (Serrano 2016). La teoría de los espacios mentales establece una relación entre el punto de vista o conceptualización que

\footnotetext{
2 Para una explicación más detallada de la prominencia cognitiva del referente de la segunda persona objetivadora, véase Aijón Oliva (2019: 192-197); Aijón Oliva y Serrano (2014: 174); Serrano (2013: 182).
} 
ofrece la cognición y el significado que un elemento lingüístico pueda representar (Sweetser 2012: 5, 17). Puesto que las estructuras lingüísticas son un espejo de la cognición, el modelo de espacios mentales analiza la relación entre las conexiones cognitivas y el lenguaje o discurso (Sweetser y Fauconnier 1996: 3). Como representación conceptual ideal, el espacio mental enmarca el dominio cognitivo sobre el que se proyecta el significado (Sweetser 2012: 3) y traslada el discurso a un espacio distinto del espacio básico predeterminado por el aquí y ahora (Croft y Cruse 2004: 33). Este dominio cognitivo se encuentra detrás del escenario y, si se activa, introduce un nuevo enfoque para que una estructura lingüística pueda interpretarse de una forma concreta. Los espacios mentales se crean, establecen o se evocan a través de distintos elementos lingüísticos, que se denominan constructores de espacios mentales (Dirven y Radden 2007: 31). Como bien señala Pascual:

Los constructores de espacios pueden describirse metafóricamente como gafas de color que se colocan encima de la nariz del ente conceptualizador motivando así una visión concreta de una entidad, hecho, situación o relación. Los espacios mentales pueden pues entenderse como escenarios cuyos elementos se conceptualizan en relación con distintos filtros cromáticos (2012: 154).

La aplicación de un filtro cognitivo es la definición más aproximada de la función que cumplen los constructores de espacios mentales en la interpretación de la segunda persona objetivadora. Los elementos temporales (cuando, si, etc.), además del uso de tiempos verbales imperfectivos como el presente de indicativo (el cual covaría con esta forma de modo categórico, v. Serrano 2013: 187), filtran un enfoque de un espacio cognitivo de continuidad o indefinitud temporal, el cual permite que la segunda persona se interprete como inclusiva del hablante y también de otras distintas a él (Serrano 2021b). Dicho de otro modo, la función que adquieren los espacios mentales en la creación del significado objetivador es el de la creación de un marco cognitivo (por ende, discursivo-pragmático) apropiado para que sea posible la interpretación objetivadora de la segunda persona tú. Marcadores temporales como cuando o si promueven que la referencia de la segunda persona aluda a un conjunto indefinido y genérico de destinatarios, porque esa referencia deja de ser precisa (deíctica) y se torna en continua y constante (objetivadora). Véase el ejemplo (10) anterior, en el que se puede comprobar que el adverbio cuando introduce tal marco temporal, que induce a que la interpretación de la segunda persona sea objetivadora.

Además, si bien la inclusión del hablante es constante en este uso (Aijón Oliva 2019: 192), la de otras personas es variable y gradual, y en ello los constructores de espacios mentales adoptan un rol esencial. Cuando la segunda persona objetivadora se construye simplemente con el presente de indicativo, pero sin marcadores temporales, el valor que adquiere es el que 
consideramos genuinamente objetivador (Serrano 2021a: 55). Este valor es el que surge cuando el participante desea expresar experiencias personales, de tal forma que el uso de la segunda persona es simplemente un recurso para mitigar la subjetividad que conllevaría el uso de la primera. Por lo tanto, la referencia que incluye es la de la primera persona, aunque ello no significa que en dicha referencia no se involucre o incluya a un posible interlocutor o audiencia, especialmente en los textos que nos ocupan.

En el siguiente ejemplo (11), la referencia a la que alude la segunda persona es el propio hablante, quien relata una experiencia propia. De hecho, en la primera parte del enunciado, a través del sintagma «mi vida», se hace patente que la persona está hablando de ella misma.

(11) Mi vida siempre había sido superdulce, aunque siempre luchando y trabajando mucho. Pero sí, era un camino de rosas hasta que se ha convertido en uno de piedras. Sin embargo, aprendes a ir de puntillas y a ver las rosas al final del camino. (CIE $<$ Object20-972>)

En cambio, la presencia de constructores de espacios mentales temporales hace que la referencia a otras personas incluya un espectro más amplio de ellas: además del propio participante, puede ser un grupo más o menos concreto de otras personas, o incluso el conjunto de la humanidad. Esto sucede porque los marcadores temporales introducen una continuidad temporal que hace que la referencia se perciba como constante y fija, por lo tanto, extrapolable a todo el conjunto de los destinatarios. Mediante la presencia de adverbios como si o cuando (entre otros elementos temporales) el contenido se presenta como desprovisto de consideraciones exclusivas o personales, pero también como general, compartido o de interés para cualquier individuo, porque se concibe como constante o que se repite.

Por ello, hemos considerado que el significado de objetivación es gradual y al que aporta esta variante lo hemos denominado objetivador + (Serrano 2021a: 55) ${ }^{3}$. Tal significado se aprecia en el ejemplo (12).

(12) Si te paras a pensarlo, nos toca la lotería todos los días: cuando encuentras esa falda por la que llevas suspirando todo el invierno, rebajada al $70 \%$ o cuando encuentras el móvil dentro del bolso a la primera. Y también cuando le dan buenos resultados en el médico a alguien que quieres (CIE $<$ Object20-937>)

El significado objetivador y objetivador + de la segunda persona objetivadora tú está presente en cualquier tipo de texto, tanto conversacional como

\footnotetext{
${ }^{3}$ En Serrano (2021a) se desarrolla la investigación sobre la función de los marcadores temporales en la delimitación de los valores graduales objetivador y objetivador + de la segunda persona objetivadora tú.
} 
de los medios de comunicación (Aijón Oliva 2019; Serrano 2013, 2021a; González Abrante 2020), y por supuesto, en los textos publicitarios, los cuales constituirán el objeto de estudio de esta investigación.

\section{Metodología y CORPus}

Se utilizarán los textos del Corpus Interaccional del Español, un corpus inédito que comprende textos de español contemporáneo de diversa naturaleza. Está estructurado y organizado en cuatro grandes secciones según las características de dichos textos: conversacionales, programas de televisión y radio autonómicas, redes sociales y textos publicitarios. Esta última sección consta de la transliteración de 594 mensajes publicitarios obtenidos en distintos medios orales y escritos: televisión, radio, YouTube, prensa (periódicos y revistas) y redes sociales. Esta sección contiene 45080 palabras. De ella hemos obtenido un total de 317 casos de segunda persona objetivadora $(t u ́)$ tanto en función de sujeto como de objeto. Estos se codificaron individualmente según los factores que se relacionan a continuación.

a) Función de sujeto o de objeto.

b) Variante expresa u omitida de la segunda persona del singular tú.

c) Constructores de espacio mental: ausencia de espacio mental, cuan$d o$, si, otros.

d) Tipo de anuncio: oral, escrito, spot publicitario. Se realiza una distinción entre anuncios orales, escritos y, por otra parte, spots publicitarios porque estos últimos suelen ser más extensos y presentan unas características textuales diferentes, tanto de naturaleza oral como escrita.

e) Ámbito comunicativo. Se han considerado los siguientes ámbitos comunicativos, entendidos como conjunto de textos que aparecen en un soporte oral o escrito determinado.

- Revistas: Se incluyen revistas denominadas femeninas o con una audiencia previsiblemente femenina, pues normalmente su contenido está, en principio, dirigido a este tipo de audiencia. Ejemplos de revistas femeninas son Elle, Vogue, Telva, Hola, etc.

- Televisión: Se incluyen anuncios y spots publicitarios orales emitidos en este soporte.

- YouTube: Se incluyen vídeos de anuncios y spots publicitarios orales emitidos en este soporte.

- Redes sociales: Se incluyen anuncios y spots publicitarios escritos en Facebook, Twitter e Instagram. 
Por lo que respecta al análisis cuantitativo, este se realizó mediante el método de cálculo de frecuencias normalizadas, también denominado variable absoluta (Aijón Oliva y Serrano 2012). Este procedimiento lleva aparejada la consideración teórica de que una variante puede ser estudiada por sí misma y no como alternativa a otras, y que dicha forma crea un significado en conjunción con otros elementos de su entorno discursivo, tanto lingüísticos como contextuales. El cálculo de estas frecuencias se realizó mediante la multiplicación de los casos de segunda persona tú objetivadora por cada 10000 palabras y la división entre el número total de palabras de los textos en los que aparece, como suele hacerse convencionalmente (Aijón Oliva y Serrano 2013: 63-64). No obstante, debido a la cantidad relativamente reducida de palabras que contiene cada uno de los tipos de anuncios y de los ámbitos, para estos se utilizará el cálculo por cada 1000 palabras.

\section{ANÁlisis de la SEgUnda PERSONA tÚ ObJETIVAdORA EN los teXtos PUBLICITARIOS}

Tras una primera cuantificación de la presencia de la segunda persona tú en el conjunto de los mensajes publicitarios del corpus (594) (45080 palabras), hemos obtenido 117 anuncios que contenían uno o varios casos de segunda persona tú con significado de objetivación (que incluyen los dos valores: objetivador y objetivador + ), de tal modo que se ha extraído la cantidad de 317 ejemplos.

TABLA 1. Casos y frecuencia normalizada de la segunda persona objetivadora tú en los mensajes publicitarios

\begin{tabular}{cc}
\hline $\begin{array}{c}\text { Casos de segunda persona tú } \\
\text { objetivadora }\end{array}$ & $\begin{array}{c}\text { Frecuencia normalizada } \\
\text { por cada } \mathbf{1 0 0 0 0} \text { palabras }\end{array}$ \\
\hline 317 & 70,3 \\
\hline
\end{tabular}

A la vista de estos datos, podemos concluir que esta forma no es demasiado frecuente en los textos publicitarios. Como era de esperar, el significado de la segunda persona predominante es el deíctico, como una vía pretendidamente eficaz para involucrar y persuadir al consumidor (véanse ejemplos (7) y (8)). Sin embargo, no por ello el significado objetivador deja de ser una estrategia de persuasión importante en el discurso publicitario. 
La cuantificación de las variantes de esta forma según las funciones de sujeto y objeto en el conjunto de ejemplos de segunda persona objetivadora (317 casos/21 476 palabras) indica que la primera es claramente más frecuente que la de objeto (Tabla 2). La función de sujeto es la que proporciona la referencia primaria de los participantes; es, por lo tanto, una función más agentiva y que involucra otras cuestiones como la animacidad y la definitud del referente (Aijón Oliva 2019: 47; Aijón Oliva y Serrano 2013: 73-74). Por ello, tiende a ser la función que predomina en cualquier tipo de texto.

TABLA 2. Casos y frecuencias normalizadas de la función de sujeto y objeto de la segunda persona tú objetivadora en sus dos valores graduales

\begin{tabular}{ccc}
\cline { 2 - 3 } & Casos & $\begin{array}{c}\text { Frecuencia normalizada } \\
\text { por cada } \mathbf{1 0 0 0 0} \text { palabras }\end{array}$ \\
\hline Función de sujeto & 178 & 82,8 \\
Función de objeto & 139 & 64,7 \\
\hline
\end{tabular}

Asimismo, siguiendo la tendencia de otras investigaciones sobre esta persona gramatical (Aijón Oliva 2019: cap. 3; Aijón Oliva y Serrano 2013: cap. 3; Serrano y Aijón Oliva 2014a: 234), las variantes omitidas de ambas funciones son claramente las más frecuentes (Tabla 3).

TABLA 3. Casos y frecuencias normalizadas de las variantes expresa y omitida de la segunda persona tú objetivadora en sus dos valores graduales

\begin{tabular}{ccc}
\cline { 2 - 3 } & Casos & $\begin{array}{c}\text { Frecuencia normalizada } \\
\text { por cada 10 000 palabras }\end{array}$ \\
\hline Variantes omitidas (sujeto y objeto) & 304 & 141,5 \\
Variantes expresas (sujeto y objeto) & 13 & 0,6 \\
\hline
\end{tabular}

Podemos concluir, por tanto, que el mensaje publicitario prototípico en el que aparece la forma objeto de estudio viene construido en formas de sujeto omitido, como el ejemplo (13).

(13) No solo corres con las piernas, corres con el corazón y corres con el alma. Decathlon. (CIE $<$ Adv2021-47>)

Como indicábamos anteriormente, el significado objetivador es gradual, y este viene determinado por la presencia de los constructores de espacios 
mentales, los cuales sitúan la referencia de la segunda persona en un ámbito temporal imperfectivo, indefinido y de continuidad ilimitada ${ }^{4}$.

Los constructores de espacios mentales son un conjunto específico de marcadores temporales, especialmente cuando y si, aunque también una cantidad bastante heterogénea y amplia de ellos actúan como tales. En la tabla 4 se presentan los casos y las frecuencias de cada constructor de espacio mental. Estas siguen la tendencia de trabajos anteriores (Serrano 2021a: 49-52), pues la frecuencia de la ausencia de constructores de espacios mentales supera la de su presencia, y, del mismo modo, cuando resultó ser el más frecuente, seguido de la categoría otros y de si.

TABLA 4. Casos y frecuencias normalizadas de los constructores de espacios mentales que coaparecen con la segunda persona tú objetivadora

\begin{tabular}{ccc}
\hline $\begin{array}{c}\text { Constructor } \\
\text { de espacios mentales }\end{array}$ & Casos & $\begin{array}{c}\text { Frecuencia normalizada } \\
\text { por cada 10 000 palabras }\end{array}$ \\
\hline Ausencia & 188 & 101,4 \\
Cuando & 49 & 26,4 \\
Otros & 44 & 23,7 \\
Si & 36 & 19,4 \\
\hline Total & $\mathbf{3 1 7}$ & $\mathbf{2 1 4 7 6}$ palabras \\
\hline
\end{tabular}

Los resultados indican que los mensajes publicitarios con segunda persona objetivadora tienden a construirse sin constructores de espacios mentales, lo cual quiere decir que adquieren el valor de objetivación prototípico o genuino, aquel que se utiliza generalmente para la formulación de experiencias personales (Serrano 2021a: 55). Esto no resulta sorprendente, pues es muy habitual que en los mensajes publicitarios la función persuasiva se escenifique mediante la experiencia personal de un participante secundario sobre el producto. Sin embargo, para evitar la ambigüedad referencial entre el significado objetivador y el deíctico, es necesario que exista evidencia de que el participante secundario es el que habla o el que escribe. El ejemplo (13) supra es una muestra de ello; un participante en el anuncio (de tipo oral) emite ese enunciado hablando por sí mismo, expresando su

\footnotetext{
${ }^{4}$ Para la correcta interpretación de las tablas, los cálculos que siguen deben entenderse del modo siguiente: la ausencia de espacios mentales se corresponde con la variante objetivadora y la presencia se corresponde con la objetivadora + .
} 
propia experiencia sobre un deporte, sin que haya ninguna otra persona presente en el anuncio, con lo cual la interpretación de la segunda persona como objetivadora es innegable.

En el siguiente anuncio escrito (14), el mensaje se presenta como verbalizado por una mujer cuya imagen se muestra. Con ello, los beneficios de un tratamiento cosmético se interpretan como la narración de su experiencia personal con el producto.

(14) La vida en la ciudad es intensa, excitante, divertida, pero te deja poco tiempo para cuidarte. Además, las agresiones ambientales estresan y debilitan tu piel, provocando su envejecimiento prematuro. AGE PREVENTION es la línea de cuidado facial de COMODINES con tratamientos que aportan lo que tu piel necesita a partir de los 30 . Con ellos previenes y combates la pérdida de vitalidad, la aparición de arrugas y líneas de expresión, manteniendo la piel joven, suave y luminosa. Gracias al exclusivo escudo protector URBAN PRO-TECH SPF15 que incorporan los tratamientos diarios COMODYNES, además de tratar, blindas tu piel frente a la contaminación, los cambios bruscos de temperatura y la radiación solar.

$(\mathrm{CIE}<\mathrm{Adv} 2021-81>)$

Con todo, en algunos casos no es fácil establecer si el referente de la segunda persona puede interpretarse como objetivador o como deíctico. En el siguiente mensaje escrito (15), pese a que también se muestra a una mujer que presta su voz al anuncio, la referencia podría considerarse ambigua: puede estar indexando a las lectoras del soporte en el que aparece, o bien puede estar incluyéndose ella misma como parte del conjunto de féminas.

(15) Seguridad en ti misma es saber que gustarte te hace aún más guapa. Ausonia. (CIE $<\operatorname{Adv} 2021-93>)$

$\mathrm{Al}$ tratarse de interacciones ficticias y diseñadas para cumplir con el propósito comunicativo de la persuasión, esta posible ambigüedad referencial se hace más evidente en el discurso publicitario que en otros contextos comunicativos. No obstante, cabe pensar que la intención persuasiva que conlleva la publicidad a menudo parte de la «experiencia personal» del publicitante o de cualquier personaje secundario para llevarla a cabo, y que con ella se pretende captar al consumidor. En el siguiente ejemplo (16), todas las formas de segunda persona participan de esta posible ambigüedad, pues incluso la variante expresa tú puede estar refiriéndose de algún modo al participante emisor y a la audiencia de forma conjunta.

(16) Un psicólogo no te proporcionará fórmulas mágicas, ni hará que en tu vida ocurran milagros, tampoco te solucionará el problema, sino que será el soporte para que tú mismo seas el que lo hagas. Un psicólogo te 
ayudará a que encuentres tus recursos, los cuales la mayoría de las veces, se encuentran guardados en tu interior o quizás, tengas que aprenderlos para iniciar ese cambio.

La posible ambigüedad se reduce, o incluso se elimina, cuando el mensaje se construye con los constructores de espacios mentales. Estos filtran cognitivamente la referencia en un ámbito referencial difuso, indefinido y continuo, lo cual permite la interpretación de segunda persona del singular tú como una amalgama referencial entre la primera y la segunda; esto es, contribuyen a la creación del significado objetivador. También en continuación con los hallazgos obtenidos en investigaciones previas (Serrano 2013: 185-187; 2021a: 52), es cuando el constructor de espacio de objetivación más frecuente. Las expresiones temporales tienen la propiedad de configurar espacialmente el tiempo de distinto modo (Haspelmath 1997: 1, 140-141). Así pues, cuando es un adverbio temporal que funciona como elemento iterativo o cuantificador universal, cuyo significado se podría parafrasear como siempre que/cada vez que, con lo cual conceptualiza el tiempo como frecuencia, sobre todo cuando se acompaña de tiempos no perfectivos (NGLE 2010: $§ 23.13 m$.). Ello lo hace especialmente útil para construir un espacio mental de indefinitud y continuidad. Este adverbio y la segunda persona $t u$ crean un significado de naturaleza normativa, constante y regular que se presupone compartido por la audiencia. En los mensajes publicitarios, la introducción de este adverbio proporciona una validez universal al producto o idea anunciado. Esta indefinitud y continuidad es, por lo tanto, muy apta para persuadir a la audiencia; se presupone que quien habla establece un punto de conexión personal con dicha audiencia para hacerle creer que ese contenido es suyo también y que va a serlo de forma inevitable.

El siguiente fragmento de un spot publicitario (17), promovido por el Ministerio de Sanidad, utiliza la primera persona del plural inclusiva al principio y al final de este («somos peleones» / «la fuerza con la que vamos a salir»). El resto está formulado en segunda persona objetivadora, introducida por cuando en dos ocasiones y con otros elementos temporales (después de tantos días, ahora, entonces). Como se observa, la voz publicitante, que actúa como participante primario, invita a adherirse al optimismo y a la superación en la situación de pandemia acaecida en el año 2020, involucra a la audiencia y la hace partícipe de una actitud que, en virtud del creador de espacio mental cuando y de la segunda persona tú, se interpreta como necesaria o indudablemente compartida o común.

(17) ... Somos peleones, aquí no somos de rendirnos y cuando peleas $\backslash$ sacas lo mejor de ti mismo\después de tantos días ya solo piensas en ganar en fijarte en las cosas que empiezan a cambiar esas son tus pequeñas victorias $\backslash$ y te das cuenta de que eres más fuerte de lo que pensabas $\backslash$ solo que 
ahora lo sabes $\backslash$ y es cuando te sientes preparado para decir: allá voy entonces abres la puerta $\backslash$ y empiezas a moverte/ y esa es nuestra fuerza $\backslash$ la fuerza con la que vamos a salir $\backslash$ (CIE $<$ Adv2021-183>)

Asimismo, en (18) y (19) la persuasión para lograr una acción se realiza mediante la misma estrategia. Se establece un punto de vista discontinuo que permite interpretar el contenido como de interés o alcance universal.

(18) Cuando pones el corazón en lo que haces, sientes auténtico Bienestar $(\mathrm{CIE}<\mathrm{Adv} 2021-141>)$

(19) No eres tú cuando tienes hambre. Snickers (CIE<Adv2021-134>)

Dicho punto de vista se puede construir también con otros elementos de variada naturaleza; la mayor parte de ellos son elementos temporales, pero también actúan como tales otros adverbios y frases adverbiales, entre ellos: después, siempre, jamás, nunca, todavía, luego, entonces, al principio, hace muchos años, un día, en primer lugar, los primeros dos o tres días, cada paso, unas veces, a veces, muchas veces, desde que, etc. (véase también Serrano 2021a: 52). Cualquiera de estos elementos funciona de modo similar al adverbio cuando. En el siguiente anuncio (20), la primera frase este verano sitúa la referencia en un punto concreto en el tiempo, pero el adverbio donde desvincula esta referencia de cualquier punto temporal concreto. De hecho, las dos formulaciones de segunda persona del primer enunciado pueden interpretarse como objetivadoras y generan un contenido de instrucción necesaria, al que le siguen varias estructuras exhortativas, ya claramente deícticas.

(20) Este verano no es donde vayas, es lo que vayas a hacer allí. Ven a Decathlon. Inspírate con nuestros productos o déjate aconsejar por nuestros deportistas. (CIE<Adv2021-177>)

La amplia gama de elementos que pueden incluirse en la categoría otros explica su elevada frecuencia en el conjunto de los mensajes publicitarios.

Por último, el adverbio condicional subordinante si actúa también como un constructor de espacio mental que enmarca el enunciado en un marco de hipotésis-consecuencia y, con ello, sitúa la interpretación en ese contexto de continuidad e indefinitud que favorece el significado objetivador de la segunda persona tú. De hecho, tanto si como cuando son adverbios que indican la regularidad de una acción, con lo cual son especialmente útiles para introducir la noción de genericidad (Montolío 1999: 3727), la cual está estrechamente relacionada con la de objetivación cognitiva. Véanse los ejemplos (21) y (22).

(21) Si puedes imaginarlo, puedes lograrlo. Si puedes soñarlo, puedes hacerlo realidad. (CIE<Adv2021-235>) 
(22) Si tienes alarma, puede ser el finde perfecto fuera de casa. Si no tienes, puede ser el fin de tu tele, tu bici, tus joyas [...] ( $\mathrm{CIE}<\mathrm{Adv} 2021-39>$ )

Una vez determinados los valores que aporta cada constructor de espacio mental en la interpretación de la segunda persona tú con significado objetivador, se examinará su uso en los distintos tipos de texto y ámbitos comunicativos en los que aparecen los mensajes publicitarios.

TABLA 5. Casos y frecuencias normalizadas de los constructores de espacios mentales según el tipo de texto del anuncio publicitario

\begin{tabular}{|c|c|c|c|c|c|c|}
\hline $\begin{array}{c}\text { Constructor } \\
\text { de espacios } \\
\text { mentales }\end{array}$ & $\begin{array}{c}\text { Casos en } \\
\text { anuncios } \\
\text { escritos } \\
(9825 \\
\text { palabras })\end{array}$ & $\begin{array}{c}\text { Frec. } \\
1000 \\
\text { palabras }^{5}\end{array}$ & $\begin{array}{c}\text { Casos en } \\
\text { anuncios } \\
\text { orales } \\
(6875 \\
\text { palabras })\end{array}$ & $\begin{array}{c}\text { Frec. } \\
1000 \\
\text { palabras }\end{array}$ & $\begin{array}{c}\text { Casos en } \\
\text { spots } \\
\text { publicitarios } \\
(4776 \\
\text { palabras })\end{array}$ & $\begin{array}{c}\text { Frec. } \\
1000 \\
\text { palabras }\end{array}$ \\
\hline Ausencia & 80 & 8,1 & 33 & 4,8 & 75 & 15,7 \\
\hline Cuando & 15 & 1,5 & 2 & 0,3 & 32 & 6,7 \\
\hline Otros & 10 & 1,01 & 20 & 2,9 & 14 & 2,9 \\
\hline Si & 24 & 2,4 & 5 & 0,7 & 7 & 1,5 \\
\hline
\end{tabular}

Como se aprecia claramente en la tabla, en todos los tipos de anuncio (escritos, orales o spots publicitarios) predomina la ausencia de constructores de espacios mentales, lo cual confirma de nuevo que la segunda persona objetivadora se puede interpretar sin constructores de espacios mentales y es la que da lugar al significado propiamente objetivador (Serrano 2021a: 55). Por lo tanto, en el discurso publicitario, estos resultados son indicativos de que la persuasión se formaliza de forma más efectiva no solo mediante la inclusión del participante que habla (ya sea un participante primario o ya sea uno secundario), sino cuando este aporta su experiencia personal sobre el producto a partir del uso de la segunda persona del singular objetivadora. En el siguiente fragmento de un spot publicitario (23), una participante anónima que publicita un producto cosmético en la plataforma YouTube utiliza la primera persona me funciona, pero posteriormente continúa compartiendo su experiencia mediante la segunda del singular objetivadora: «no te van a salir granitos»; «va muy bien para desmaquillarte»;

\footnotetext{
${ }^{5}$ Debido al escaso número total de palabras de cada tipo de texto, hemos realizado el cálculo por cada 1000 palabras.
} 
«te cuesta cinco euros». La indexación de la audiencia se hace evidente también mediante el uso de la segunda persona del plural, que obviamente ya son deícticos (veis, os digo, tenéis).

(23) La verdad que me funciona muy bien\este tiene 250 mililitros y está fabricado en Sevilla $\backslash$ súper bien por Mercadona $\backslash$ este $\backslash$ al contrario que el de coco $\backslash$ este no es comedogénico $\backslash$ no $t e$ van a salir granitos $\backslash$ y va muy bien para desmaquillarte porque además es un aceite muy muy hidratante $\backslash$ el aguacate es un fruto que tiene mucho aceite $\backslash$ con lo cual este aceite es muy nutritivo/ y es muy hidratante $\backslash$ este::: muy hidratante $\backslash$ veis aunque me he puesto el aceite::: pero no queda para nada la mano aceitosa $\backslash$ es como que se absorbe muy bien $\backslash$ por eso sirve muy bien para desmaquillar $\backslash t e$ cuesta cinco euros $\backslash$ pero os digo yo que esto cunde una barbaridad $\backslash$ con lo cual con este bote tenéis para rato $\backslash(\mathrm{CIE}<\operatorname{Adv} 2021-172>$ )

La alta frecuencia de este uso con ausencia de constructores de espacios mentales en los spots publicitarios se explica porque estos son textos de mayor extensión, donde la referencia conjunta al propio hablante y al interlocutor o audiencia suele estar activada y suele ser fácilmente identificable, con lo cual el referente de segunda persona objetivadora no se presta a la ambigüedad. No obstante, si bien en los spots publicitarios tiende a predominar la variante objetivadora o la que expresa experiencias personales sobre el producto o idea publicitado (y ello explica la mayor ausencia de constructores de espacios mentales), es posible que, dada su extensión, este alterne con segmentos generalizadores o de alcance universal; esto es, puede coaparecer con la variante con significado objetivador + . Ejemplo de ello es el fragmento del spot siguiente (24) que finaliza con un enunciado de este tipo, introducido por a veces.

... El aspecto de la crema es un poco raro $\backslash$ lo acabo de ver ahora y el olor es es también muy fuerte $\backslash$ es como muy fuerte $\backslash$ igual no te lo pones y no $\backslash$ y es más normal $\backslash$ pero:: pero bueno \no lo vuelvo a repetir no lo sé $\backslash$ no lo sé \no :::tengo que probar con lo que sigue [...] ya sabéis que hay cosas que a veces de una marca te gusta una cosa pero las demás cosas pues no te gusta $\backslash(\mathrm{CIE}<\mathrm{Adv} 2021-223>)$

En los anuncios escritos (que incluyen también spots publicitarios), la frecuencia de ausencia de constructores de espacios mentales se explica a partir de su elevado nivel de planificación; se presupone que los anuncios de esta naturaleza están sometidos a una planificación en la que la posible ambigüedad no debería producirse, más aún cuando está en juego la capacidad persuasiva del mensaje. Por ejemplo, en (25), los dos primeros enunciados contienen casos de segunda persona objetivadora; sin embargo, el último: «nunca lo dudes», al estar introducido por un adverbio negativo, puede deshacer la referencia objetivadora de esta persona y quizás la 
de la siguiente «viajar te lleva a alguna parte». Sin embargo, el entorno tipográfico del mensaje publicitario escrito (imágenes de personas realizando viajes), puede contribuir a que se mantenga la referencia objetivadora, en este caso apta para promover la persuasión.

(25) Por eso se puede afirmar que viajar te hace mejor persona. Te renueva, te da nuevos bríos y hace posible que tu vida se llene de más color y magia. Nunca lo dudes: viajar siempre te lleva a alguna parte. (CIE $<\operatorname{Adv2021-~}$ $172>)$

Si bien lo mismo debería aplicarse a los anuncios orales, lo cierto es que la presencia de los constructores de espacios mentales es también inferior a la de los textos escritos y a la de los spots publicitarios. En este tipo de anuncios, sin embargo, el uso de la voz ayuda a que la interpretación de la segunda persona vaya en el camino deseado. Normalmente, este valor surge, al igual que en los spots publicitarios, a partir de la experiencia de un participante secundario (anónimo o no) que habla de las bondades del producto, ya sea a la audiencia, ya sea a otro participante presente en el anuncio. Tal es el caso del siguiente anuncio oral (26), en el que una voz va recreando diferentes situaciones reales en las que esa voz representa la queja de que «a alguien le falta algo» con distintos interlocutores que aparecen en el vídeo. Al final, surge el eslogan, que contiene la segunda persona objetivadora en función de objeto te.

(26) Somos asî parece que siempre nos falta algo \no sabemos muy bien el qué/ pero es un algo \como a ese novio del colegio al que le faltaba un no sé qué no\noไno\no\ eso no es\ hasta las suegras lo sueltan: -Aquí también falta algo!/ por eso te presentamos ALGO \aunque al nombre también le faltaba y le hemos llamado AJONESAl

Una mayonesa con ese algo más $\backslash$ que $t e$ da vidilla. (CIE<Adv2021-144>)

Por lo que respecta al uso de cuando, las frecuencias indican que está presente sobre todo en los spots publicitarios. Esto se explica por las razones señaladas; al ser textos de una extensión mayor que los anuncios, la posibilidad de que aparezcan constructores de espacios mentales puede aumentar. Lo mismo puede decirse de la categoría otros, que alcanza la misma frecuencia en los anuncios orales. En cambio, si solo tiene cierta representación en los textos escritos. Como se aprecia claramente en el ejemplo (27), la unidad si construye el espacio mental que hace posible que el contenido se presente como de validez universal. La segunda persona objetivadora, introducida por si, construye el significado objetivador + .

(27) Si no vas, no llegas. Si no empiezas, no acabas. Si no eres, no estás. Si no sueñas, no creas. Si no observas, no ves. Si no enseñas, no aprendes. Si no sientes, no vives. (CIE<Adv2021-252>) 
La distribución de las frecuencias de los constructores de espacio mental según el ámbito comunicativo arroja resultados similares, pues en todos ellos predomina su ausencia.

TABLA 6. Casos y frecuencias normalizadas

de los constructores de espacios mentales según el ámbito comunicativo del anuncio publicitario

\begin{tabular}{|c|c|c|c|c|}
\hline & \multicolumn{4}{|c|}{ Constructor de espacios mentales } \\
\hline & Ausencia & Cuando & Otros & Si \\
\hline $\begin{array}{l}\text { Casos en revistas } \\
\text { (4659 palabras) }\end{array}$ & 42 & 8 & 4 & 5 \\
\hline Frec. 1000 palabras & 9,01 & 1,7 & 0,9 & 1,1 \\
\hline $\begin{array}{l}\text { Casos en televisión } \\
\text { (4492 palabras) }\end{array}$ & 33 & 14 & 18 & 5 \\
\hline Frec. 1000 palabras & 7,3 & 3,1 & 0,1 & 1,1 \\
\hline $\begin{array}{l}\text { Casos en YouTube } \\
\text { (7 159 palabras) }\end{array}$ & 75 & 20 & 16 & 7 \\
\hline Frec. 1000 palabras & 10,4 & 2,7 & 2,2 & 0,9 \\
\hline $\begin{array}{l}\text { Casos en redes sociales } \\
\text { (5 166 palabras) }\end{array}$ & 38 & 7 & 6 & 19 \\
\hline Frec. 1000 palabras & 7,3 & 1,3 & 1,2 & 3,6 \\
\hline
\end{tabular}

En coincidencia con los resultados de las tablas 4 y 5 , los casos procedentes de YouTube son los que presentan una frecuencia ligeramente superior de ausencia de constructores, pues es de esta plataforma de donde se ha extraído la mayor parte de los ejemplos de spots publicitarios. Su frecuencia en los ejemplos de revistas es también coherente con los datos de los anuncios escritos, que en conjunto presentaron la misma tendencia de ausencia de constructores.

Por lo que respecta a la frecuencia de cuando, se observa una ligera tendencia a aparecer en los ejemplos de la televisión y en los de YouTube. En el caso de esta plataforma, como se explicó anteriormente, algunos vídeos, por su estructura, pueden contener otros marcadores temporales que contribuyen a la construcción de la objetivación. Los ejemplos de la televisión, contrariamente, suelen ser más cortos y con tendencia al eslogan, razón por la que cuando garantizaría que la referencia se interprete de forma objetivadora y no deíctica, tal y como se aprecia en los ejemplos (28) y (29). 
(28) Cuando abres una caja roja de Nestlé, a la vida se le escapa una sonrisa. (CIE<Adv2021-59>)

(29) Porque cuando te sientes vital, te sientes imparable. (CIE<Adv2021-131>)

Asimismo, estos resultados indican que la mayor frecuencia del constructor si en el conjunto de los anuncios escritos reflejada en la tabla 5 era debida al número de casos obtenidos de las redes sociales, puesto que en este nuevo cálculo la frecuencia de si alcanza un 3,6. En este tipo de texto, además de publicidad sobre un producto concreto, son muy habituales las publicaciones sobre ideas o consejos de tipo emocional, normalmente encaminados a adherirse a algún grupo, página o sociedad, tal y como se observa en (30).

(30) Algo va mal si estás cansado, si te invade la tristeza, la desilusión o el desencanto y si sientes que lo que haces por la otra persona es una carga cuando no debería serlo. (CIE<Adv2021-276>)

Quien ha elaborado este texto utiliza la segunda persona, pero el constructor $s i$ hace posible que el consejo o instrucción se proyecte también sobre el participante que lo ha escrito.

\section{Conclusiones}

La segunda persona con significado objetivador $(t u ́)$ en el discurso publicitario, si bien no parece ser un recurso excesivamente frecuente, es una estrategia al servicio de la persuasión que define este tipo de texto. El objetivo primordial de este discurso es alertar emocionalmente a los consumidores para involucrarlos en el mensaje publicitario y con ello lograr que adquieran un producto determinado o se sumen a una idea. En el cumplimiento de ese objetivo, la inclusión del participante que habla o escribe en la referencia de la segunda persona es determinante; al fusionar su propia referencia con la de la audiencia o interlocutor, estos se perciben como incluidos en el contenido, el cual, al mismo tiempo, se transforma en desubjetivador, ya que constituye un tránsito del dominio de la primera persona hacia la segunda. La objetividad que conlleva este fenómeno puede verse como un apoyo a la función persuasiva que define el discurso publicitario; si el contenido se percibe como alejado de la esfera personal del participante, esto es, más objetivo, es previsible que conduzca de forma más efectiva al consumo.

La aproximación cognitiva a este fenómeno ofrece una explicación para la correcta interpretación objetivadora de la segunda persona. Los marcadores temporales cuando, si y otros construyen un espacio cognitivo de con- 
tinuidad y de indefinitud ilimitada que permite enfocar la referencia de la segunda persona como de alcance general o universal. Sin embargo, la cuantificación realizada en la presente investigación redunda en los resultados obtenidos en las precedentes; esta persona se construye más frecuentemente en ausencia de constructores de espacios mentales, lo cual indica que la segunda persona tú con valor genuinamente objetivador es la variante más frecuentemente utilizada en el discurso publicitario. Esto se explica porque en este tipo de discurso resulta esencial que el publicitante, representado como participante primario o secundario (sobre todo, secundario, presente en el anuncio), muestre su experiencia personal para validar el producto que se publicita; de este modo, conseguirá persuadir a la audiencia y establecer una conexión emocional con ella. Por su parte, el significado objetivador + , generado mediante la presencia de los constructores de espacios mentales, aparece en aquellas situaciones comunicativas más proclives a expresar este tipo de contenidos, como lo son algunos anuncios de tipo oral y los spots publicitarios, así como los mensajes publicitarios que se obtienen de las redes sociales. Este segundo valor, sin menoscabo de su valor persuasivo, implica al consumidor potencial de forma más genérica y universal, esto es, no representa solo la experiencia del publicitante, sino que la extiende de forma más clara a la segunda persona. La propia asociación de la segunda persona tú objetivadora a hechos habituales o recurrentes dota al contenido de valor general, de tal forma que lo presenta como verdad comúnmente aceptada.

Nuevamente, podemos concluir la importancia de adoptar un enfoque cognitivo en el estudio de la variación morfosintáctica. En este caso, se ha comprobado que la función que desempeñan los constructores de espacios mentales como filtros que proporcionan una percepción del evento comunicativo resulta crucial para establecer el significado. Los constructores permiten delimitar de modo formal y funcional una distinción gradual en el significado objetivador de la segunda persona, pero, además, demuestran la existencia de cambios en el significado asociados a la forma; esto es, en última instancia, estos resultados insisten, una vez más, en la ausencia de sinonimia entre las variantes morfosintácticas. 


\section{BIBLIOGRAFÍA}

AjJón Oliva, Miguel Ángel (2009): «Tú y usted como estrategias de estilo y persuasión en la comunicación publicitaria», Tonos Digital 18. <http://www.tonos digital.es/ojs/index.php/tonos/article/view/338/261>.

- (2019): Constructing Us. The First and Second Persons in Spanish Media Discourse, Berlín: Mouton de Gruyter.

- (2020): «It Can Be Us or You. The Desubjectification of Viewpoint through Person Choice in Spanish Oral and Written Media Discourse», Journal of Pragmatics 163, 4-17.

- y María José SERRANo (2012): «Towards a Comprehensive View of Variation in Language: The Absolute Variable», Language \& Communication 32, 80-94.

- y - (2013): Style in Syntax. Investigating Variation in Spanish Pronoun Subjects, Berna: Peter Lang.

— y - (2014): «Moving towards the Realm of the Other: Second-person Objectivization in Spanish Media Discourse», Language Sciences 45, 173-188.

Armstrong, Scott (2010): Persuasive Advertising. Evidence-based Principles, Nueva York: Palgrave.

Barron, Anne (2012): Public Information Messages. A Contrastive Genre Analysis of State-citizen Communication, Ámsterdam: John Benjamins Publishing.

BeINHAUER, Werner (1968): El español coloquial, Madrid: Gredos.

CAMERon, Richard (1993): «Ambiguous Agreement, Functional Compensation and Nonspecific tú in the Spanish of San Juan in Puerto Rico, and Madrid, Spain», Language Variation and Change 5, 305-334.

ColEman, Linda (1990): «The Language of Advertising», Journal of Pragmatics $14 / 1,137-145$.

Croft, William y Allan D. Cruse (2004): Cognitive Linguistics, Cambridge: Cambridge University Press.

CoOK, Guy (2001): The Discourse of Advertising, London: Routledge.

DiRVEN, René y Günter RADDEN (2007): Cognitive English Grammar (Vol. 2), Ámsterdam: John Benjamins Publishing.

DyER, Gillian (1982): Advertising as Communication, Londres: Routledge.

ENRÍQUEZ, Emilia (1984): El pronombre personal sujeto en la lengua española hablada en Madrid, Madrid: Centro Superior de Investigaciones Científicas.

Evans, Vyvyan y Melanie Green (2006): Cognitive linguistics: An Introduction, Lawrence Erlbaum Associates Publishers.

FAUCONNIER, Gilles (1985 [1994]): Mental Spaces. Aspects of Meaning Construction in Natural Languages, Cambridge: University Press.

— (1997): Mappings in Thought and Language, Cambridge: University Press.

Fernández Soriano, Olga y Susana TÁboas Baylín (1999): «Construcciones impersonales no reflejas». En Ignacio Bosque y Violeta Demonte (dirs.), Gramática descriptiva de la lengua española, Madrid: Espasa-Calpe, 1723-1778. 
Ferraz Martínez, A. (1993): El lenguaje de la publicidad, Madrid: Arco/Libros.

Fuentes Rodríguez, Catalina y Esperanza Alcaide Lara (2002): Mecanismos lingüisticos de la persuasión, Madrid: Arco/Libros.

GILI GAYA, Samuel (1976): Curso superior de sintaxis española, Barcelona: Biblograf.

GODDARD, Angela (1998): The Language of Advertising, Londres: Routledge.

GONZÁLEZ ABRANTE, Zaida (2020): «La segunda persona objetivadora ( $t u ́$ ) en las redes sociales y revistas: datos de textos escritos», Borealis. An International Journal of Hispanic Linguistics 9/2, 209-231.

GuIRADO, Kristel (2011): «La alternancia tú uno impersonal en el habla de Caracas», Lingüistica 26, 24-54.

Haspelmath, Martin (1997): From Space to Time. Temporal Adverbials in the World's Language, Múnich: Lincom Europa.

Hermeren, Lund (1999): English for Sale: A Study of the Language of Advertising, Lund: Lund University Press.

Hernández Toribio, María Isabel (2006): El poder de la palabra en la publicidad de radio, Barcelona: Octaedro.

Hernanz Carbó, María Luisa (1990): «En torno a los sujetos arbitrarios: la segunda persona del singular». En Violeta Demonte y Beatriz Garza Cuarón (eds.), Estudios de lingüistica de España y México, México: El Colegio de México, 151-178.

Hidalgo Navarro, Antonio (1996-1997): «Sobre los mecanismos de impersonalización en el español coloquial: el tú impersonal», Estudios de Lingüistica de la Universidad de Alicante 11, 163-176.

Hugo Rojas, Evelyn (2011): «Las formas de segunda persona singular como estrategias evidenciales», Revista de Lingüistica Teórica y Aplicada 49/1, 143-167.

KANY, Charles (1969): Sintaxis hispanoamericana, Madrid: Gredos.

Kitagawa, Chisato y Adrienne Lehrer (1990): «Impersonal Uses of Personal Pronouns», Journal of Pragmatics 14, 739-759.

LABERGE, Suzanne y Gillian SANkoff (1979): «Any thing you can do». En Talmy Givón (ed.), Discourse and Syntax, Nueva York/San Francisco: Academic Press, 419-440.

LEECH, Geoffrey (1966): English in Advertising. A Linguistic Study of Advertising in Great Britain, Londres: Longman.

Montolío, Estrella (1999): «Las construcciones condicionales». En Ignacio Bosque y Violeta Demonte (dirs.), Gramática descriptiva de la lengua española, Madrid: Espasa-Calpe, 3643-3738.

NGLE: ReAl ACAdemia Española y Asociación de ACAdemias de la Lengua EsPaNOLA (2010): Nueva gramática de la lengua española, Madrid: Espasa-Calpe.

PASCUAL, Esther (2012): «Los espacios mentales y la integración conceptual». En Iraide Ibarretxe Antuñano y Javier Valenzuela Manzanares (coords.), Lingüistica cognitiva, Barcelona: Anthropos, 147-166.

Romero Gualda, María Victoria (1996): El español en los medios de comunicación, Madrid: Arco/Libros.

SECO, Manuel (1989): Gramática esencial del español, Madrid: Espasa-Calpe.

SERRANO, María José (2013): «El pronombre tú como recurso objetivador en español: variación textual y discursiva», Borealis. An International Journal of Hispanic Linguistics 2/1, 179-197. 
- (2016): «La variación sintáctica». En Javier Gutiérrez-Rexach (ed.), Enciclopedia de Lingüistica Hispánica, Londres: Routledge, 809-821.

- (2020): «Shaping Socio-communicative Identities in Interaction: The Variable Usage of the Second-person Object usted(es)», International Review of Pragmatics $12,80-106$.

- (2021a): «Gradual Objectivivity. Variation in the Use of the Objectivizing Second-person Singular tú in Spanish», Journal of Pragmatics 176, 44-62.

- (2021b): «Variation in Society: The Periphrases tener/haber que + infinitive by Sex/gender of Participants in Spanish», Pragmatics and Society (aceptado para su publicación).

— y Miguel Ángel Aujón Oliva (2012): «Cuando tú eres yo: la inespecificidad referencial de tú como objetivación del discurso», Nueva Revista de Filología Hispánica 60, 541-563.

- y - (2014a): «Discourse objectivization, social variation and style in the use of Spanish tú», Folia Linguistica 48/1, 225-254.

- y - (2014b): "Seguimos con la actualidad... The Omission of Nosotros 'We' across Spanish Media Genres», Discourse \& Communication 7/4, 409-433.

- y María Isabel Hernández Toribio (2015): «¿Porque tú lo vales o porque lo vales?». Variación de la segunda persona tú en los mensajes publicitarios», Ibérica 30, 105-128.

SweEtSer, Eve (2012): «Introduction: Viewpoint and Perspective in Language and Gesture, from the Ground Down». En Barbara Dancygier y Eve Sweetser (eds.), Viewpoint in Language. A Multimodal Perspective, Nueva York: Cambridge University Press, 1-24.

— y Gilles Fauconnier (1996): «Cognitive Links and Domains: Basic Aspects of Mental Spaces Theory». En Gilles Fauconnier y Eve Sweetser (eds.), Spaces, Worlds, and Grammar, Chicago/Londres: The University of Chicago Press, $1-28$.

Siewierska, Anna (2004): Person, Cambridge: Cambridge University Press.

VÁzQuez Medel, Miguel Ángel (1993): «Introducción al análisis del discurso publicitario», Questiones publicitarias 1/1, 42-54.

VestergaARD, Torben y Kim SCHRODER (1985): The Language of Advertising, Oxford: Blackwell.

ZACHER, Robert (1967): Advertising Techniques and Management, Homewood: Richard Irwin. 